\title{
Pentablock Copolymer from Tetracomponent Monomer Mixture Using a Switchable Dizinc Catalyst
}

\author{
Thomas T. D. Chen, Yunqing Zhu, and Charlotte K. Williams*(0) \\ Department of Chemistry, Chemistry Research Laboratory, 12 Mansfield Road, Oxford OX1 3TA, U.K. \\ Supporting Information
}

ABSTRACT: Well-defined pentablock copolymers are prepared using a single catalyst, in one pot, from four different monomers: anhydride, epoxide, lactone, and $\mathrm{CO}_{2}$. The dizinc catalyst bridges three distinct polymerization cycles and performs a double switch in polymerization mechanism to produce pentablock copolymers. The new materials are hydroxyl-telechelic and are efficiently postfunctionalized to introduce polar and nonpolar side-chains.

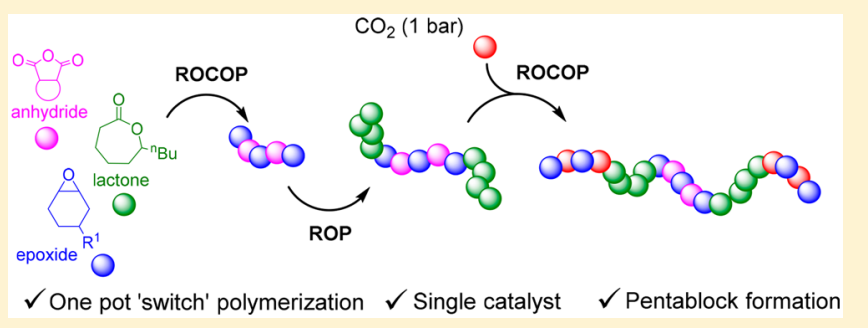

$\mathrm{N}$ ature synthesizes molecules with exquisite product selectivity. ${ }^{1}$ Synthetic chemistry struggles with selective reactivity from mixtures, although catalysis could solve the problem if directing of reaction pathways were better understood. Such selective catalysis could simplify process chemistry by obviating intermediate isolations and purifications, thereby reducing energy usage and costs. In polymerization catalysis, such selectivity may also deliver well-defined copolymer sequences. ${ }^{2}$ The most widely investigated block polymers have $\mathrm{AB}$ or $\mathrm{ABA}$ structures, and applications include thermoplastic elastomers, toughened plastics, and drug delivery. More complex block structures are difficult to prepare (e.g., the number of potential block order variations are proportional to the factorial of the number of monomers used) but are expected to show superior properties. ${ }^{3}$ Multiblock copolymers are usually prepared using macroinitiators, followed by intermediate isolations and purifications, or by multiple timed monomer additions. ${ }^{4-8}$ A simpler strategy is to switch a single catalyst between different polymerization cycles, thus allowing the enchainment of a much greater variety of monomers. For example, Kamigaito and co-workers pioneered a trithiocarbonate initiator that switched between controlled radical acrylate and cationic vinyl ether polymerizations. ${ }^{9}$ Aoshima and co-workers applied $\mathrm{B}\left(\mathrm{C}_{6} \mathrm{~F}_{3}\right)_{3}$ to catalyze both cationic polymerization of vinyl ethers and epoxides. ${ }^{10}$

We target sustainable block copolymers featuring degradable ester/carbonate linkages and utilizing $\mathrm{CO}_{2} \cdot{ }^{11}$ These materials may be prepared using lactone ring-opening polymerization (ROP) to form poly(lactones) or epoxide $/ \mathrm{CO}_{2}$ (anhydride) ring-opening copolymerization (ROCOP) to form alternating polyesters/polycarbonates (PE/PC). ${ }^{12-14}$ While terpolymerizations using a single mechanism are well-known, ${ }^{15,16}$ polymerizations applying both mechanisms are less investigated. ${ }^{17-19}$ Tandem catalysis, applied by Darensbourg and $\mathrm{Wu}$, enabled linking of the catalytic cycles to form ABA block polymers. $^{20,21}$ We reported a single catalyst active using mixtures of epoxides $/ \mathrm{CO}_{2} /$ lactones, switching between
ROCOP and ROP, to produce block copolymers. ${ }^{19,22,23}$ Subsequently, $\mathrm{CO}_{2}$ was replaced for anhydrides to form $\mathrm{ABA}$ block copolyesters. ${ }^{24,25}$ In 2017, Rieger and co-workers reported another dizinc catalyst selective for three-component monomer mixtures. ${ }^{26}$ Recently, we reported how to switch a commercial Cr-Salen catalyst so as to prepare ABA triblocks (again from three component mixtures). ${ }^{27}$ Until now, all studies applied only mixtures of three different monomers limiting the resulting sequence to $\mathrm{AB}(\mathrm{A})$. A previous $\mathrm{DFT}$ study of three-component mixtures suggested that a clear future target should be to investigate mixtures of four monomers, accessing three different catalytic cycles. ${ }^{23}$ Here, we experimentally demonstrate such a selective catalysis using mixtures of four monomers and a single catalyst (Scheme 1).

Catalyst 1, $\left[\mathrm{LZn}_{2} \mathrm{Ph}_{2}\right]$, was activated using trans-1,2cyclohexanediol (CHD) to form the zinc alkoxide complex in situ (see Scheme S1 for the activation of $\mathbf{1}$ and Scheme S2 for ROP and ROCOP mechanisms). ${ }^{28}$ This catalyst is selected because it is highly controlled, yielding only hydroxyl-telechelic polymers with monomodal molar mass distributions in each separate polymerization. ${ }^{24,28}$ The latter is paramount since most other ROCOP catalysts result in bimodal molar mass distributions with differing end-groups. ${ }^{20-22,24,29,30}$ Such bimodality prevents selective multiblock formation as it causes contamination by lower block sequences (i.e., $\mathrm{AB} / \mathrm{ABC} / \mathrm{ABCB}$ type). ${ }^{31,32}$ The catalyst system was reacted with four monomers (phthalic anhydride (PA)/cyclohexene oxide $(\mathrm{CHO}) / \varepsilon$-decalactone $\left.(\mathrm{DL}) / \mathrm{CO}_{2}\right)$, at $0.1 \mathrm{~mol} \%$ catalyst loading, and $100{ }^{\circ} \mathrm{C}$ under $\mathrm{CO}_{2}$ ( 1 bar pressure). DL was chosen as the model lactone as it affords a secondary metal alkoxide propagating species when ring-opened which results in well-balanced kinetics and resistance to transesterification; both features are expected to be important for effective

Received: June 12, 2018

Revised: June 19, 2018

Published: July 11, 2018 
Scheme 1. Four Monomer Selective Polymerization, Using $\left[\mathrm{LZn}_{2} \mathrm{Ph}_{2}\right]$ (1)/trans-1,2-Cyclohexanediol (CHD) Catalytic System (See Scheme S1 for in Situ Activation of the Catalyst)

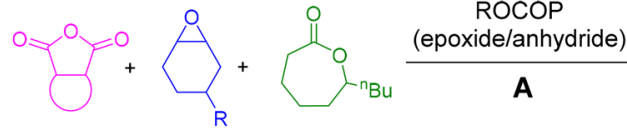

anhydride epoxide $\varepsilon$-decalactone

(DL)

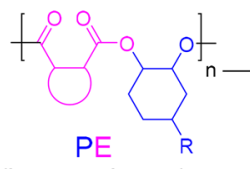

(homopolymer)
ROP

(lactone)
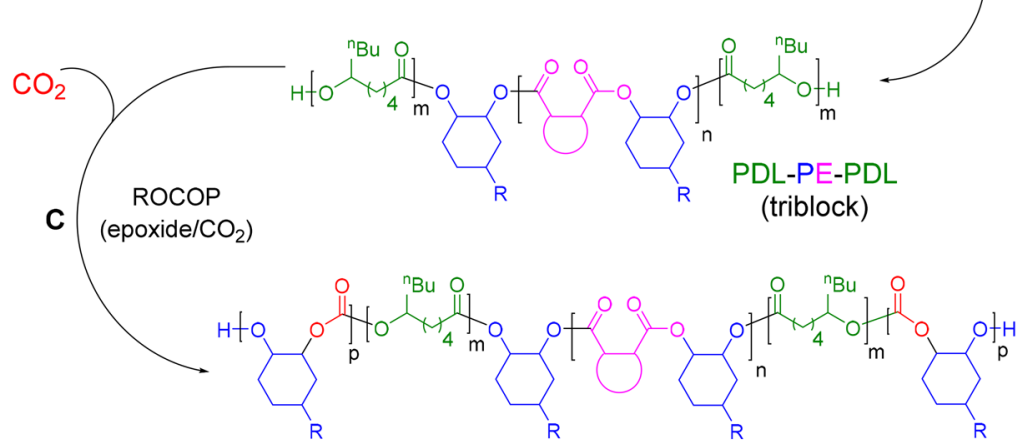

PC-PDL-PE-PDL-PC (pentablock)
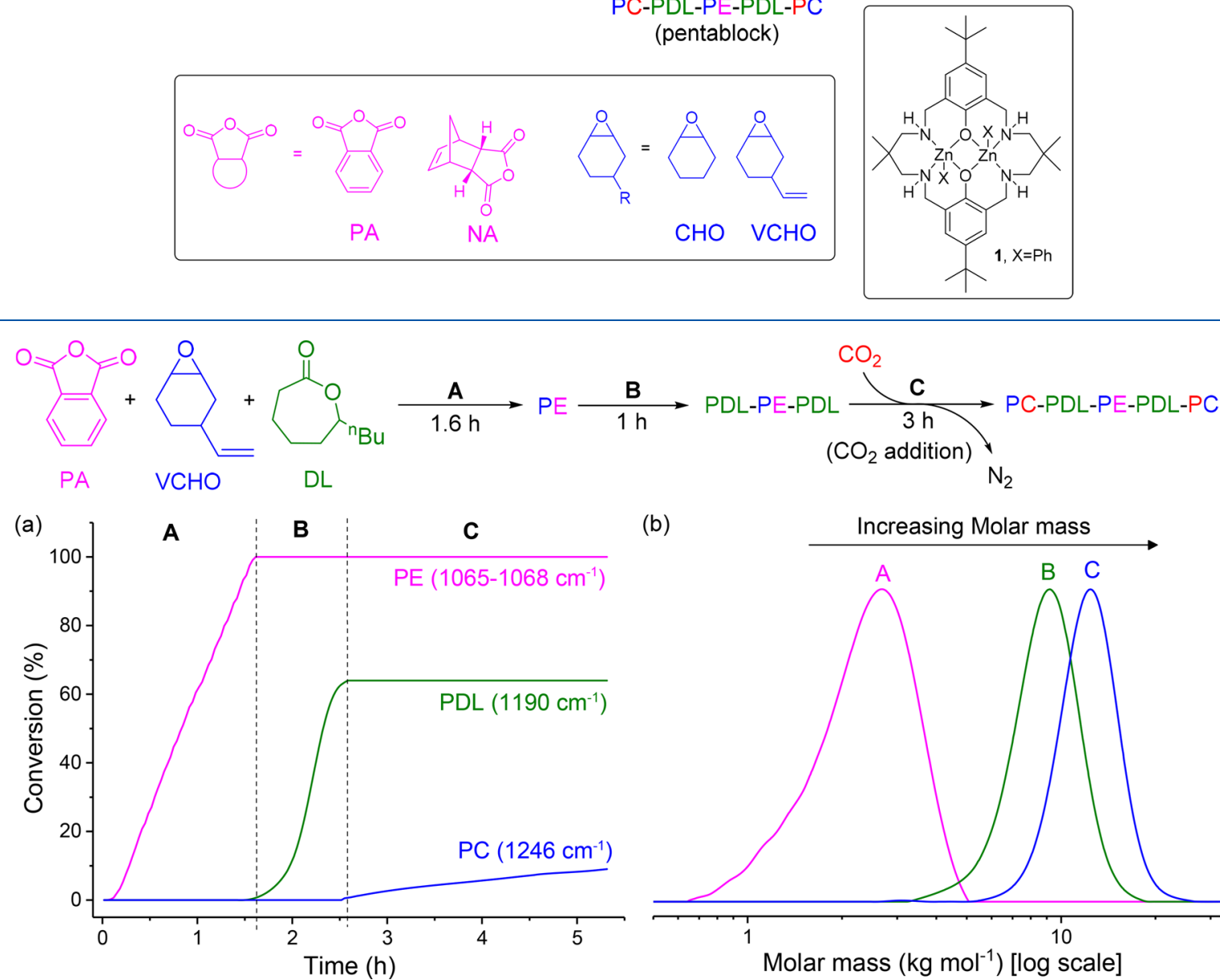

(b)

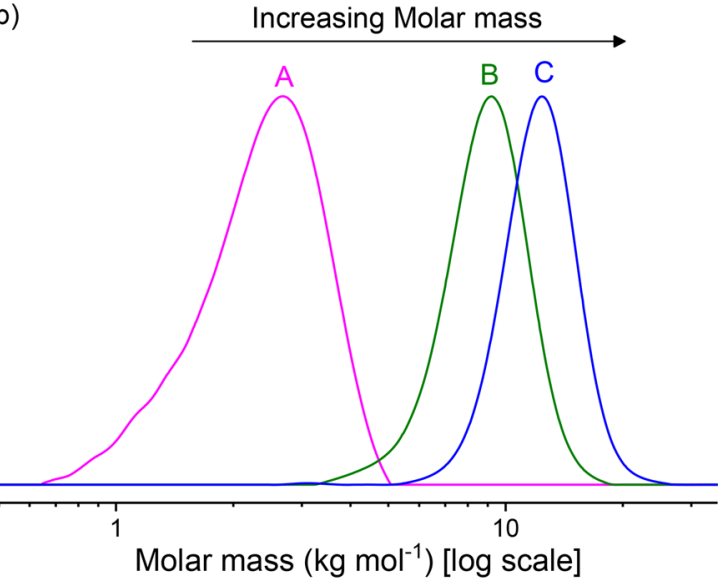

Figure 1. Polymerization of tetracomponent monomer mixture: $\mathrm{PA} / \mathrm{VCHO} / \mathrm{DL} / \mathrm{CO}_{2} ;[1] /[\mathrm{CHD}] /[\mathrm{PA}] /[\mathrm{DL}] /[\mathrm{VCHO}]=1 / 4 / 50 / 200 / 1000$, $100{ }^{\circ} \mathrm{C}$, (A, B) $\mathrm{N}_{2}$ and (C) $\mathrm{CO}_{2}, 1$ bar. (a) Conversion vs time plot illustrating block formation: A (anhydride/epoxide ROCOP), B (lactone ROP), and $\mathrm{C}$ (epoxide/ $\mathrm{CO}_{2}$ ROCOP). Conversions obtained using in situ ATR-IR spectroscopy and calibrated using ${ }^{1} \mathrm{H}$ NMR spectroscopy (Figure S4). (b) SEC traces illustrating the increase in $M_{\mathrm{n}}$ and retention of $Ð$ as the polymerization progresses (calibrated with narrow dispersity polystyrene standards).

polymerization control. ${ }^{33}$ The reaction yielded only the PCPE-PC triblock formed by ROCOP. To drive DL ROP, the $\mathrm{CO}_{2}$ was completely removed, but poly $(\varepsilon$-decalactone) (PDL) block formation was not observed even after $20 \mathrm{~h}$ (Figure S3).
Rather, the trans-cyclic carbonate byproduct slowly evolved. The formation of trans-cyclohexene oxide indicates that chain backbiting occurred from the metal alkoxide intermediate (Scheme S3). ${ }^{32}$ Thus, while it is clear that the metal alkoxide 
Table 1. Four Monomer Polymerizations Using Anhydride, Epoxide, Lactone, and $\mathrm{CO}_{2}{ }^{a}$

\begin{tabular}{|c|c|c|c|c|c|c|c|c|c|c|}
\hline & \multirow[b]{2}{*}{ monomer combination } & \multicolumn{3}{|c|}{ conversion $^{b}(\%)$} & \multicolumn{3}{|c|}{$M_{\mathrm{n}}\left(\mathrm{kg} \mathrm{mol}{ }^{-1}\right)[Đ]^{c}$} & \multicolumn{3}{|c|}{$\operatorname{TOF}^{d}\left(\mathrm{~h}^{-1}\right)$} \\
\hline & & anhydride & lactone & epoxide $^{e}$ & A & B & $\mathrm{C}$ & PE ROCOP & PDL ROP & PC ROCOP \\
\hline P1 & $\mathrm{PA} / \mathrm{CHO} / \mathrm{DL} / \mathrm{CO}_{2}$ & $>99$ & 69 & 11 & $1.7[1.12]$ & $7.5[1.15]$ & $8.8[1.11]$ & 27 & 160 & 6 \\
\hline P2 & $\mathrm{PA} / \mathrm{VCHO} / \mathrm{DL} / \mathrm{CO}_{2}$ & $>99$ & 64 & 14 & $2.1[1.16]$ & $8.4[1.08]$ & $12.1[1.05]$ & 32 & 128 & 30 \\
\hline P3 & $\mathrm{NA} / \mathrm{CHO} / \mathrm{DL} / \mathrm{CO}_{2}$ & $>99$ & 79 & 12 & $1.4[1.15]$ & $6.5[1.12]$ & $8.4[1.08]$ & 4 & 126 & 9 \\
\hline P4 & $\mathrm{NA} / \mathrm{VCHO} / \mathrm{DL} / \mathrm{CO}_{2}$ & $>99$ & 80 & 16 & $1.5[1.15]$ & $7.1[1.11]$ & $10.2[1.14]$ & 8 & 160 & 44 \\
\hline
\end{tabular}

${ }^{a}[1] /[\mathrm{CHD}] /[$ anhydride $] /[$ lactone $] /[$ epoxide $]=1 / 4 / 50 / 200 / 1000,100{ }^{\circ} \mathrm{C},(\mathrm{A}, \mathrm{B}) \mathrm{N}_{2}$ and $(\mathrm{C}) \mathrm{CO}_{2}, 1$ bar. PA, NA, DL, CHO, and VCHO denote phthalic anhydride, cis-5-norbornene-endo-2,3-dicarboxylic anhydride, $\varepsilon$-decalactone, cyclohexene oxide, and 4-vinyl-1-cyclohexene 1,2epoxide, respectively. ${ }^{b}$ Obtained from ${ }^{1} \mathrm{H}$ NMR spectra (Figures S4 and S26-28). ${ }^{c}$ Measured by SEC, calibrated with polystyrene (Figures S23S25). ${ }^{d}$ Calculated from molar monomer conversion per mole of catalyst per hour. ${ }^{e}$ Note: the apparently low overall conversions for epoxide are due to its dual use as monomer and solvent.

intermediate is accessed, the rate of backbiting reaction is proposed to occur faster than the initiation of DL ROP. This problem was overcome by a simple change in monomer addition order. First, the catalyst system was reacted with PA/ $\mathrm{CHO} / \mathrm{DL}$, forming the desired PDL-PE-PDL copolyester (Scheme 1A,B). Thereafter, the $\mathrm{N}_{2}$ atmosphere was replaced with 1 bar pressure of $\mathrm{CO}_{2}$, resulting in selective PC-PDLPE-PDL-PC pentablock formation.

The tetracomponent mixture polymerization was generalized to include a functionalized epoxide (vinylcyclohexene oxide, $\mathrm{VCHO}$ ). Throughout the polymerization, aliquots were analyzed using ${ }^{1} \mathrm{H}$ NMR spectroscopy to quantify the monomer conversions against an internal standard (mesitylene) (Figure S4) and by SEC to quantify molar masses (Figure $1 \mathrm{~b}$ ). The initial ${ }^{1} \mathrm{H}$ NMR spectrum showed complete consumption of anhydride (8.02 and $7.90 \mathrm{ppm})$ and concurrent formation of polyester (7.66 and $7.50 \mathrm{ppm})$. The alternating copolymerization with $\mathrm{VCHO}$ was also confirmed (5.35 and $5.22 \mathrm{ppm}$ ). PDL blocks were only observed once all anhydride was consumed (4.84 ppm). The sequential block formation is also observed using in situ ATR-IR spectroscopy, where formation of PE $\left(1065-1068 \mathrm{~cm}^{-1}\right)$ is followed by PDL $\left(1190 \mathrm{~cm}^{-1}\right)$. To demonstrate the high selectivity, $\mathrm{CO}_{2}$ was added before full $\mathrm{DL}$ conversion and an immediate switch from DL ROP to $\mathrm{VCHO} / \mathrm{CO}_{2}$ ROCOP occurred, as evidenced by the PC formation $\left(1246 \mathrm{~cm}^{-1}\right)$. The ${ }^{1} \mathrm{H}$ NMR spectrum at this stage also showed the growth of a signal at $4.76 \mathrm{ppm}$ assigned to PC (Figure S4). All IR and ${ }^{1} \mathrm{H}$ NMR resonances were assigned by comparison with resonances observed in the constituent polymers (Figures S5-S18).

It is important to note that high polymer conversions are achieved in each block, but as the epoxide is applied as both reagent and solvent its conversion appears lower. Nonetheless, the epoxide conversion is fully consistent with the formation of perfectly alternating PE and PC blocks. SEC analyses show a clear increase in molar mass with retention of narrow dispersity $(\nexists<1.17)$ (Figure 1b). The catalyst maintains its activity throughout polymerization, with differing rates for each stage (Table 1). ${ }^{31} \mathrm{P}\left\{{ }^{1} \mathrm{H}\right\}$ NMR spectroscopy enables characterization of chain end groups (Figure S19). ${ }^{24,34}$ During stage A peaks are observed at 146.7-146.8 ppm (assigned to PE) that shifted to a single peak for PDL (147.1 ppm) after stage B. Stage C revealed peaks consistent with PC formation (146.6$146.9 \mathrm{ppm})$. At all stages, hydroxyl end-group signals are observed. ${ }^{1} \mathrm{H}$ DOSY NMR analysis of the pentablock shows a single diffusion coefficient for all resonances, suggesting all blocks are joined (Figure S20, top). In contrast, a blend of the appropriate polymers, at related molar masses, shows three different diffusion coefficients (Figure S20, bottom). The extent of transesterification must be understood since it could scramble block structure. ${ }^{34}$ The pentablock copolymer showed no detectable transesterification: the ${ }^{13} \mathrm{C}$ NMR spectrum showed three major carbonyl peaks, corresponding to each of the three blocks; furthermore, SEC analysis reveals narrow dispersity values throughout the polymerization (Figure S21). Purification of the crude pentablocks did not change their composition; i.e., there is no significant homopolymer contamination (Figure S22). Overall, the characterization data are consistent with selective pentablock copolymer formation. This polymerization breaks new ground in proceeding with two switches between different polymerization mechanisms, specifically from epoxide/anhydride ROCOP to lactone ROP, followed by epoxide/ $\mathrm{CO}_{2}$ ROCOP.

The generality of the four monomer switchable catalysis was investigated using various anhydride/epoxide combinations (Table 1). High selectivity for pentablock formation was observed in all mixtures. The anhydride was investigated using cis-5-norbornene-endo-2,3-dicarboxylic anhydride (NA) (Table 1, P3 and P4). Both reactions occurred first through NA/ epoxide ROCOP (A), followed by DL ROP (B) and finally by epoxide/ $\mathrm{CO}_{2}$ ROCOP (C), as confirmed using in situ ATR-IR and ${ }^{1} \mathrm{H}$ NMR spectroscopy (Figures S24-S28). The SEC analyses show molar mass increases and narrow dispersity in all samples $(\nexists<1.17)$ (Figures S24 and S25). The pentablock structures were once again confirmed using a range of techniques (vide supra) (Figures S29-S35). In all reactions, even though DL ROP is the faster reaction, the slower epoxide/anhydride ROCOP occurs first (Table 1). This selectivity is proposed to arise from the zeroth-order rate dependence on the insertion of anhydride and $\mathrm{CO}_{2}$ into the zinc alkoxide intermediate (Figure 2). ${ }^{32,35}$ In addition, DFT investigations highlight the relative linkage stability as well as the overall barrier in controlling selectivity; the zinc carboxylate/carbonate intermediates (anhydride $/ \mathrm{CO}_{2}$ insertion) are significantly more stable than the alternative zinc alkoxide (lactone insertion). ${ }^{23}$ Overall, the chemistry of the zinc chain end group controls the monomer selectivity, linking the three different polymerizations and controlling monomer discrimination.

Several of the pentablock copolymers contain alkene groups that were postfunctionalized to install different side chains. Generally, functionalized lactones are difficult to synthesize and polymerize which has limited investigation of functionalized, degradable polyesters. Recently, ROCOP was used to prepare various alkene-substituted polyesters and carbonates that were later efficiently reacted without compromising the polymer backbone. ${ }^{29,36-40}$ Here, the well-known thiol-ene reaction is applied to install alkane or hydroxyalkane 


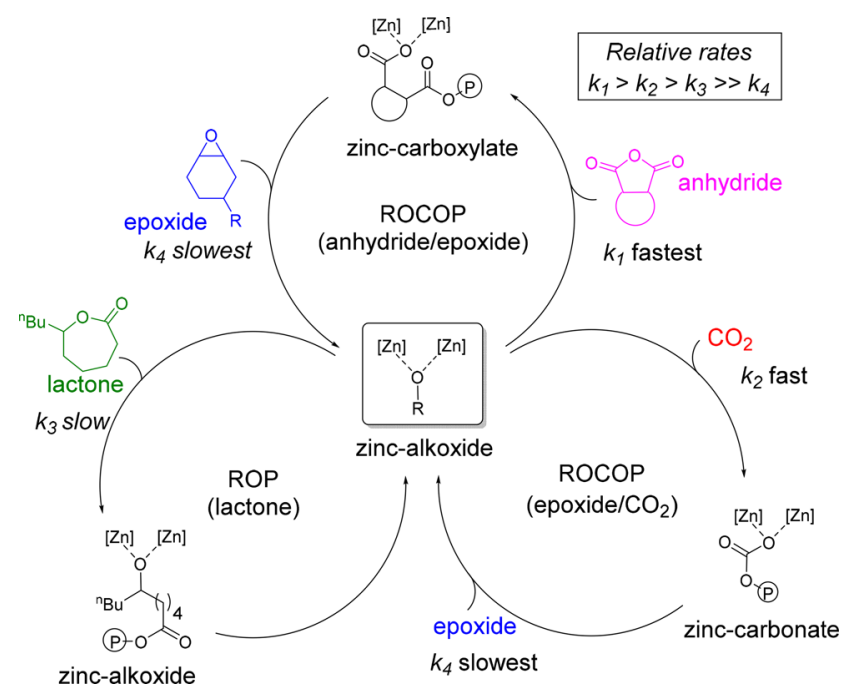

Figure 2. Proposed mechanisms occurring during polymerization of anhydride, epoxide, lactone, and $\mathrm{CO}_{2}$, highlighting the central role for the zinc alkoxide intermediate ( $\mathrm{P}=$ growing polymer chain).

substituents. ${ }^{41}$ UV-initiated reactions were performed on pentablock P2 using either 1-butanethiol or 2-mercaptoethanol (Figure 3). The reactions reached complete conversion within $2 \mathrm{~h}$, as shown by ${ }^{1} \mathrm{H}$ NMR spectroscopy (Figure S37). The double-bond resonances are consumed during the reaction (5.76 and 5.01-5.09 ppm), but all the other resonances remain unchanged (Figure S22). New resonances confirm attachment of the butyl thioether $(2.51,0.90 \mathrm{ppm})$ or hydroxyl ethyl thioether groups $(3.72,2.71$, and $2.55 \mathrm{ppm})$. The functionalized polymers show slightly higher molar masses compared to the starting pentablocks with retention of monomodal, narrow dispersity; these findings suggest the polymer backbone remains intact. The pentablock copolymers P3 and P4 were also successfully reacted to produce analogous functionalized polymers (Table S1 and Figures S38-S41). In the case of the block copolymer P4, both internal and terminal double bonds react with the thiol reagent.

Preliminary assessment of pentablock copolymers' thermal properties indicates a single $T_{\mathrm{g}}$ for all polymers, suggesting amorphous structures with miscible blocks (Table S2). The block miscibility is tentatively attributed to the lower overall molar masses, since the propensity to undergo phase separation is directly correlated to degree of polymerization. Interestingly, the $T_{\mathrm{g}}$ was easily varied over the range -35 to 20 ${ }^{\circ} \mathrm{C}$. Moreover, the $T_{\mathrm{g}}$ can be further adjusted by side-chain functionalization: addition of hydroxyl alkyl side-chains increases the $T_{\mathrm{g}}$ (typically $>20^{\circ} \mathrm{C}$ ) compared to the precursor, presumably due to hydrogen bonding. ${ }^{42}$ The pentablocks' controllable, low $T_{\mathrm{g}}$, low molar masses, and hydroxyl-telechelic structures indicate they are suited for further development as
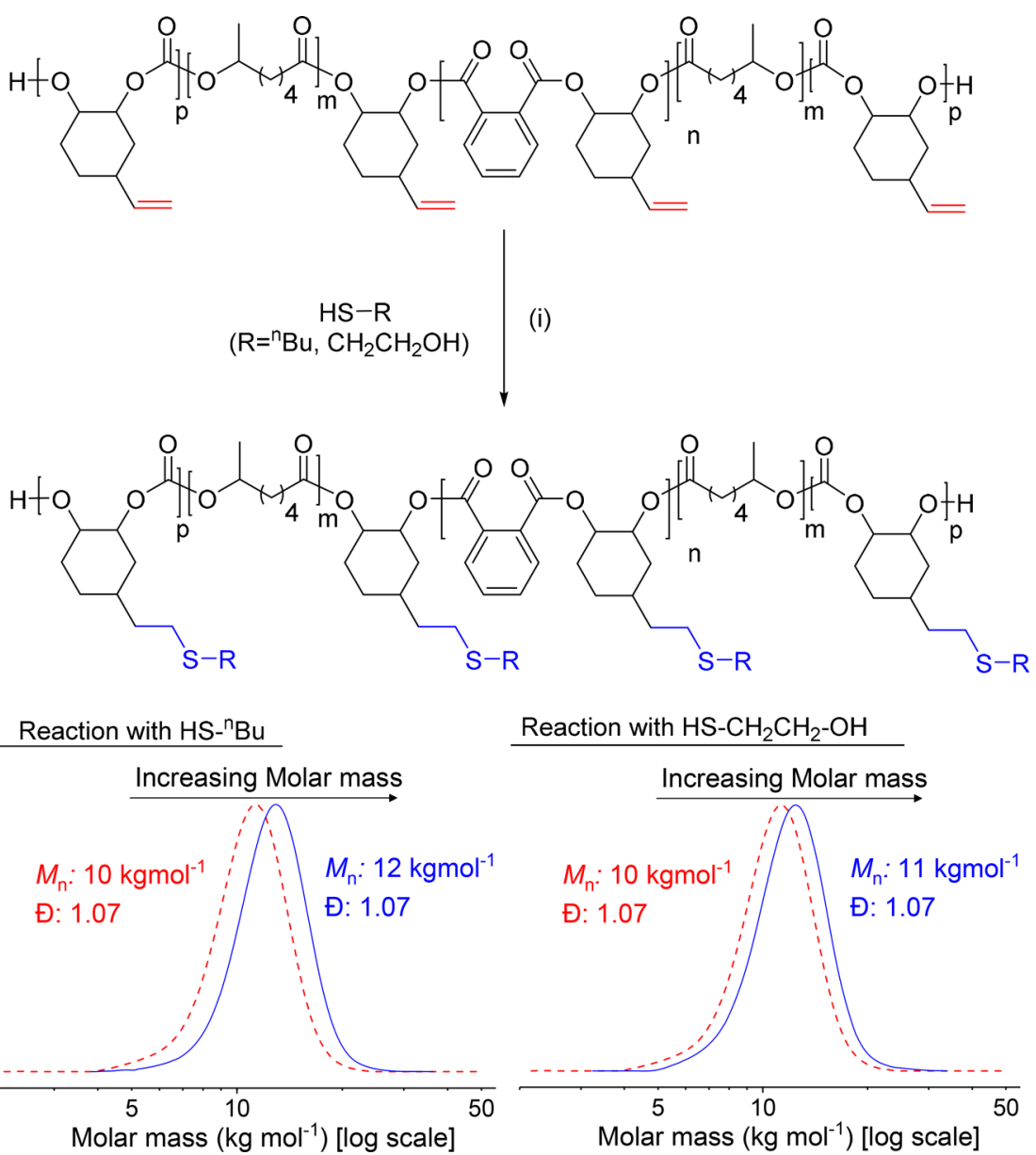

Figure 3. Thiol-ene functionalization of the pentablock copolymers. (i) 2,2-Dimethoxy-2-phenylacetophenone (DMPA, photoinitiator)/alkene/ thiol reagent $=1 / 2.5 / 10, \mathrm{UV}, 2 \mathrm{~h}, 25^{\circ} \mathrm{C}, \mathrm{CDCl}_{3}$. Dashed red and solid blue lines illustrate the molar mass distributions before and after reaction, respectively. 
polyols. Recently, polycarbonate polyols, produced using carbon dioxide ROCOP, are being commercially investigated for polyurethane manufacture; these new pentablocks significantly expand the range of carbon dioxide containing polyols. $^{43}$

In summary, switchable catalysis is demonstrated using mixtures of four monomers to selectively produce pentablock copolymers. The method applies a single catalyst in one pot to yield hydroxyl-telechelic pentablocks. Fundamentally, the catalyst accesses three different polymerization cycles and switches mechanism twice. Overall, the work demonstrates the high selectivity and complexity of block structures that can be delivered using switchable catalysis. More generally, it should be applicable to other catalysts and monomer combinations. Applications for the new pentablock polyols in areas such as semirenewable coatings, cross-linked resins, and polyurethane manufacture are recommended. ${ }^{44}$ Finally, the switchable catalysis is expected to enable variation in polymer composition, block volume fraction, and molar mass producing new thermoplastic elastomers, toughened plastics, and even functionalized medical materials.

\section{ASSOCIATED CONTENT}

\section{S Supporting Information}

The Supporting Information is available free of charge on the ACS Publications website at DOI: 10.1021/acs.macromol.8b01224.

Experimental section; Schemes S1 and S2, Figures S1S52, Tables S1 and S2 (PDF)

\section{AUTHOR INFORMATION}

\section{Corresponding Author}

*E-mail: Charlotte.williams@chem.ox.ac.uk (C.K.W.).

\section{ORCID}

Charlotte K. Williams: 0000-0002-0734-1575

\section{Notes}

The authors declare the following competing financial interest(s): C.K.W. is a director and CSO of Econic Technologies.

\section{ACKNOWLEDGMENTS}

The EPSRC (EP/L017393/1, EP/K014668/1) and EIT Climate $\mathrm{KIC}$ (project $\mathrm{EnCO}_{2} \mathrm{re}$ ) are acknowledged for funding.

\section{REFERENCES}

(1) Lutz, J. F.; Lehn, J. M.; Meijer, E. W.; Matyjaszewski, K. From precision polymers to complex materials and systems. Nat. Rev. Mater. 2016, 1, 16024.

(2) Bates, C. M.; Bates, F. S. 50th Anniversary Perspective: Block Polymers-Pure Potential. Macromolecules 2017, 50, 3-22.

(3) Bates, F. S.; Hillmyer, M. A.; Lodge, T. P.; Bates, C. M.; Delaney, K. T.; Fredrickson, G. H. Multiblock Polymers: Panacea or Pandora's Box? Science 2012, 336, 434-440.

(4) Ouchi, M.; Terashima, T.; Sawamoto, M. Transition MetalCatalyzed Living Radical Polymerization: Toward Perfection in Catalysis and Precision Polymer Synthesis. Chem. Rev. 2009, 109, 4963-5050.

(5) Matyjaszewski, K.; Tsarevsky, N. V. Nanostructured functional materials prepared by atom transfer radical polymerization. Nat. Chem. 2009, 1, 276-288.

(6) Moad, G. RAFT polymerization to form stimuli-responsive polymers. Polym. Chem. 2017, 8, 177-219.
(7) Hillmyer, M. A.; Tolman, W. B. Aliphatic Polyester Block Polymers: Renewable, Degradable, and Sustainable. Acc. Chem. Res. 2014, 47, 2390-2396.

(8) Lutz, J. F.; Ouchi, M.; Liu, D. R.; Sawamoto, M. SequenceControlled Polymers. Science 2013, 341, 1238149.

(9) Kumagai, S.; Nagai, K.; Satoh, K.; Kamigaito, M. In-Situ Direct Mechanistic Transformation from RAFT to Living Cationic Polymerization for (Meth)acrylate-Vinyl Ether Block Copolymers. Macromolecules 2010, 43, 7523-7531.

(10) Kanazawa, A.; Kanaoka, S.; Aoshima, S. Concurrent Cationic Vinyl-Addition and Ring-Opening Copolymerization Using B(C6F5) (3) as a Catalyst: Copolymerization of Vinyl Ethers and Isobutylene Oxide via Crossover Propagation Reactions. J. Am. Chem. Soc. 2013 135, 9330-9333.

(11) Zhu, Y. Q.; Romain, C.; Williams, C. K. Sustainable polymers from renewable resources. Nature 2016, 540, 354-362.

(12) Longo, J. M.; Sanford, M. J.; Coates, G. W. Ring-Opening Copolymerization of Epoxides and Cyclic Anhydrides with Discrete Metal Complexes: Structure-Property Relationships. Chem. Rev. 2016, 116, 15167-15197.

(13) Paul, S.; Zhu, Y. Q.; Romain, C.; Brooks, R.; Saini, P. K.; Williams, C. K. Ring-opening copolymerization (ROCOP): synthesis and properties of polyesters and polycarbonates. Chem. Commun. 2015, 51, 6459-6479.

(14) Guillaume, S. M.; Kirillov, E.; Sarazin, Y.; Carpentier, J. F. Beyond Stereoselectivity, Switchable Catalysis: Some of the Last Frontier Challenges in Ring-Opening Polymerization of Cyclic Esters. Chem. - Eur. J. 2015, 21, 7988-8003.

(15) Jeske, R. C.; Rowley, J. M.; Coates, G. W. Pre-rate-determining selectivity in the terpolymerization of epoxides, cyclic anhydrides, and $\mathrm{CO}_{2}$ : A one-step route to diblock copolymers. Angew. Chem., Int. Ed. 2008, 47, 6041-6044.

(16) Broderick, E. M.; Guo, N.; Vogel, C. S.; Xu, C. L.; Sutter, J.; Miller, J. T.; Meyer, K.; Mehrkhodavandi, P.; Diaconescu, P. L. Redox Control of a Ring-Opening Polymerization Catalyst. J. Am. Chem. Soc. 2011, 133, 9278-9281.

(17) Aida, T.; Sanuki, K.; Inoue, S. Well-Controlled Polymerization by Metalloporphyrin - Synthesis of Copolymer with Alternating Sequence and Regulated Molecular-Weight from Cyclic Acid Anhydride and Epoxide Catalyzed by the System of Aluminum Porphyrin Coupled with Quaternary Organic Salt. Macromolecules 1985, 18, 1049-1055.

(18) Kroger, M.; Folli, C.; Walter, O.; Doring, M. Alternating copolymerization of carbon dioxide and cyclohexene oxide and their terpolymerization with lactide catalyzed by zinc complexes of N,N ligands. Adv. Synth. Catal. 2006, 348, 1908-1918.

(19) Romain, C.; Williams, C. K. Chemoselective Polymerization Control: From Mixed-Monomer Feedstock to Copolymers. Angew. Chem., Int. Ed. 2014, 53, 1607-1610.

(20) Wu, G. P.; Darensbourg, D. J.; Lu, X. B. Tandem MetalCoordination Copolymerization and Organocatalytic Ring-Opening Polymerization via Water To Synthesize Diblock Copolymers of Styrene Oxide $/ \mathrm{CO}_{2}$ and Lactide. J. Am. Chem. Soc. 2012, 134, 17739-17745.

(21) Darensbourg, D. J.; Wu, G.-P. A One-Pot Synthesis of a Triblock Copolymer from Propylene Oxide/Carbon Dioxide and Lactide: Intermediacy of Polyol Initiators. Angew. Chem., Int. Ed. 2013, 52, 10602-10606.

(22) Paul, S.; Romain, C.; Shaw, J.; Williams, C. K. Sequence Selective Polymerization Catalysis: A New Route to ABA Block Copoly(ester-b-carbonate-b-ester). Macromolecules 2015, 48, 60476056.

(23) Romain, C.; Zhu, Y. Q.; Dingwall, P.; Paul, S.; Rzepa, H. S.; Buchard, A.; Williams, C. K. Chemoselective Polymerizations from Mixtures of Epoxide, Lactone, Anhydride, and Carbon Dioxide. J. Am. Chem. Soc. 2016, 138, 4120-4131.

(24) Zhu, Y.; Romain, C.; Williams, C. K. Selective Polymerization Catalysis: Controlling the Metal Chain End Group to Prepare Block Copolyesters. J. Am. Chem. Soc. 2015, 137, 12179-12182. 
(25) Zhu, Y.; Radlauer, M. R.; Schneiderman, D. K.; Shaffer, M. S. P.; Hillmyer, M. A.; Williams, C. K. Multiblock Polyesters Demonstrating High Elasticity and Shape Memory Effects. Macromolecules 2018, 51, 2466-2475.

(26) Kernbichl, S.; Reiter, M.; Adams, F.; Vagin, S.; Rieger, B. $\mathrm{CO}_{2-}$ Controlled One-Pot Synthesis of AB, ABA Block, and Statistical Terpolymers from beta-Butyrolactone, Epoxides, and $\mathrm{CO}_{2}$. J. Am. Chem. Soc. 2017, 139, 6787-6790.

(27) Stosser, T.; Williams, C. K. Selective Polymerization Catalysis from Monomer Mixtures: Using a Commercial Cr-Salen Catalyst To Access ABA Block Polyesters. Angew. Chem., Int. Ed. 2018, 57, 63376341.

(28) Romain, C.; Garden, J. A.; Trott, G.; Buchard, A.; White, A. J. P.; Williams, C. K. Di-Zinc-Aryl Complexes: $\mathrm{CO}_{2}$ Insertions and Applications in Polymerisation Catalysis. Chem. - Eur. J. 2017, 23, $7367-7376$.

(29) Sanford, M. J.; Van Zee, N. J.; Coates, G. W. Reversibledeactivation anionic alternating ring-opening copolymerization of epoxides and cyclic anhydrides: access to orthogonally functionalizable multiblock aliphatic polyesters. Chem. Sci. 2018, 9, 134-142.

(30) Van Zee, N. J.; Coates, G. W. Alternating Copolymerization of Propylene Oxide with Biorenewable Terpene-Based Cyclic Anhydrides: A Sustainable Route to Aliphatic Polyesters with High Glass Transition Temperatures. Angew. Chem., Int. Ed. 2015, 54, 26652668.

(31) Saini, P. K.; Fiorani, G.; Mathers, R. T.; Williams, C. K. Zinc versus Magnesium: Orthogonal Catalyst Reactivity in Selective Polymerizations of Epoxides, Bio-derived Anhydrides and Carbon Dioxide. Chem. - Eur. J. 2017, 23, 4260-4265.

(32) Jutz, F.; Buchard, A.; Kember, M. R.; Fredriksen, S. B.; Williams, C. K. Mechanistic Investigation and Reaction Kinetics of the Low-Pressure Copolymerization of Cyclohexene Oxide and Carbon Dioxide Catalyzed by a Dizinc Complex. J. Am. Chem. Soc. 2011, 133, 17395-17405.

(33) Zhu, Y. Q.; Romain, C.; Poirier, V.; Williams, C. K. Influences of a Dizinc Catalyst and Bifunctional Chain Transfer Agents on the Polymer Architecture in the Ring-Opening Polymerization of epsilonCaprolactone. Macromolecules 2015, 48, 2407-2416.

(34) Stosser, T.; Chen, T. T. D.; Zhu, Y.; Williams, C. K. 'Switch' catalysis: from monomer mixtures to sequence-controlled block copolymers. Philos. Trans. R. Soc., A 2018, 376, 20170066.

(35) Saini, P. K.; Romain, C.; Zhu, Y. Q.; Williams, C. K. Dimagnesium and zinc catalysts for the copolymerization of phthalic anhydride and cyclohexene oxide. Polym. Chem. 2014, 5, 6068-6075.

(36) Darensbourg, D. J.; Tsai, F. T. Postpolymerization Functionalization of Copolymers Produced from Carbon Dioxide and 2Vinyloxirane: Amphiphilic/Water-Soluble $\mathrm{CO}_{2}$-Based Polycarbonates. Macromolecules 2014, 47, 3806-3813.

(37) Darensbourg, D. J.; Chung, W. C.; Arp, C. J.; Tsai, F. T.; Kyran, S. J. Copolymerization and Cycloaddition Products Derived from Coupling Reactions of 1,2-Epoxy-4-cyclohexene and Carbon Dioxide. Postpolymerization Functionalization via Thiol-Ene Click Reactions. Macromolecules 2014, 47, 7347-7353.

(38) Li, C. L.; van Berkel, S.; Sablong, R. J.; Koning, C. E. Postfunctionalization of fully biobased poly(limonene carbonate)s: Synthesis, characterization and coating evaluation. Eur. Polym. J. 2016, 85, 466-477.

(39) Hauenstein, O.; Agarwal, S.; Greiner, A. Bio-based polycarbonate as synthetic toolbox. Nat. Commun. 2016, 7, 11862.

(40) Han, B.; Zhang, L.; Liu, B. Y.; Dong, X. F.; Kim, I.; Duan, Z. Y.; Theato, P. Controllable Synthesis of Stereoregular Polyesters by Organocatalytic Alternating Copolymerizations of Cyclohexene Oxide and Norbornene Anhydrides. Macromolecules 2015, 48, 3431-3437.

(41) Hoyle, C. E.; Bowman, C. N. Thiol-Ene Click Chemistry. Angew. Chem., Int. Ed. 2010, 49, 1540-1573.

(42) Cho, S.; Heo, G. S.; Khan, S.; Gonzalez, A. M.; Elsabahy, M.; Wooley, K. L. Functionalizable Hydrophilic Polycarbonate, Poly(5methyl-5-(2-hydroxypropyl)aminocarbonyl-1,3-dioxan-2-one), De- signed as a Degradable Alternative for PHPMA and PEG. Macromolecules 2015, 48, 8797-8805.

(43) von der Assen, N.; Bardow, A. Life cycle assessment of polyols for polyurethane production using $\mathrm{CO}_{2}$ as feedstock: insights from an industrial case study. Green Chem. 2014, 16, 3272-3280.

(44) Stosser, T.; Li, C. L.; Unruangsri, J.; Saini, P. K.; Sablong, R. J.; Meier, M. A. R.; Williams, C. K.; Koning, C. Bio-derived polymers for coating applications: comparing poly(limonene carbonate) and poly (cyclohexadiene carbonate). Polym. Chem. 2017, 8, 6099-6105. 